\title{
Construcción y características psicométricas de la Escala de Estrategias de Estimulación del Autoconcepto Académico
}

\author{
Karla Lobos Peña ${ }^{1}$ \\ Universidad Santo Tomás, Chile \\ Alejandro Díaz Mujica, Claudio Bustos Navarrete, Fabiola Sáez Delgado \\ Psicología Universidad de Concepción, Chile
}

\section{RESUMEN}

Se elaboró y se evaluó el comportamiento psicométrico de una escala de estrategias de estimulación del autoconcepto académico, asociada a comportamientos observados en la interacción profesor-alumno. Participaron del estudio 819 estudiantes de Eseñanza Primaria, de 16 escuelas de alta vulnerabilidad social, de la ciudad de Concepción, en Chile. La escala fue construída después de una revisión teórica, aplicación de dos pilotos con los alumnos y una validación interjueces. Los resultados del análisis factorial exploratorio indican que la escala final, con 15 preguntas, tiene una estructura de tres factores de primer orden (estrategias de capacidad, procedimientos de trabajo y de participación), que explican $66 \%$ de la varianza, con un factor de segundo orden con un omega jerárquico de 0,89 . Esta estructura es corroborada por medio de un análisis factorial confirmatorio. Se observa validez concurrente con la subescala de autoestima escolar del Inventario de Autoestima de Coopersmith.

Palabras clave: interacción profesor-alumno; autoconcepto; construcción de test; psicología educativa.

\section{RESUMO - Escala de Estratégias de Estimulação do Autoconceito Acadêmico}

Elaborou-se e avaliou-se o comportamento psicométrico de uma escala de estratégias de estimulação do autoconceito acadêmico associada a comportamentos observáveis da interação professor-aluno. O estudo contou com a participação de 819 alunos do ensino fundamental de 16 escolas de alta vulnerabilidade social na cidade chilena de Concepción. A escala foi construída após uma revisão teórica, a aplicação de dois pilotos com os alunos e uma validação interjuízes. Os resultados de uma análise exploratória indicam que a escala final, com 15 perguntas, tem uma estrutura de três fatores de primeira ordem (estratégias de capacidade, de procedimentos de trabalho e de participação), que explicam $66 \%$ da variância, com um fator de segunda ordem com um ômega hierárquico de 0,89 . Esta estrutura é corroborada por uma análise fatorial confirmatória. Observa-se validade concorrente com a subescala de autoestima escolar do Inventário de Autoestima de Coopersmith.

Palavras-chave: interação professor-aluno; autoconceito; construção de teste; psicologia educacional.

\section{ABSTRACT - Scale of strategies for stimulating Academic Self-Concept}

The objective of this study was to elaborate and evaluate behavioral psychometrics of a scale for stimulating academic self-concept strategies associated with observable teacher-student interaction behaviors. A total of 819 elementary students from 16 schools with high social vulnerability in the Chilean city of Concepción. The scale was constructed after a theoretical revision, the application of two pilot studies with the students, and an inter-judicial validation. Exploratory analysis results indicate that the final scale, with 15 questions, has a structure of three first-order factors (capacity, work procedures and participation strategies), which explain $66 \%$ of the variance, with a factor of second-order with a hierarchical omega of 0.89 . This structure is corroborated by a confirmatory factor analysis. Concurrent validity is observed with the school self-esteem subscale of Coopersmith's Self-Esteem Inventory. Keywords: teacher-student interaction; self-concept; test construction; educational psychology.

Las variables motivacionales están asociadas de manera importante a los logros académicos de los estudiantes. La evidencia muestra que la escuela influye de manera decisiva en el desarrollo de los procesos motivacionales del alumno, por lo que es posible diseñar estrategias que fomenten la motivación, mejorando así el rendimiento académico (Carbonero, Martin-Anton, Reoyo, \& Román, 2010).
Entre las variables cognitivo motivacionales, destaca el autoconcepto académico, definido como la autopercepción del alumno acerca de su competencia para enfrentar distintas actividades y tareas escolares (Marsh \& Martin, 2011). En los estudios transversales, el autoconcepto académico destaca como el factor con mayor relación con el rendimiento y suele ser el de mayor capacidad predictiva 
en estudios longitudinales (Castejón y Miñano, 2011; Coelho, Sousa, \& Figueira, 2014; Gallardo, Garfella, Sánchez, Ros \& Serra, 2008; Guay, Boivin, \& Marsh, 2003; Guay, Larose, \& Boivin, 2004; Green, Marsh, \& O'Mara, 2006; Hui-Ju, 2009; Marsh \& Martin, 2011).

Desde la década del 70 se ha estudiado la relación entre autoconcepto y rendimiento académico desde 3 enfoques distintos: 1. el rendimiento académico como causa del autoconcepto, 2. el nivel de autoconcepto como causa del grado de logro académico y 3 . enfoque integrador, que considera la influencia recíproca entre el autoconcepto y el rendimiento (González, Núñez, Glez, \& García, 1997; Marsh, 1990; Marsh \& Craven, 2006). Si bien diversas investigaciones con estudiantes confirman que existe una relación recíproca entre ambos, se observa un mayor poder predictivo del autoconcepto sobre el rendimiento académico que viceversa (Sánchez-Oliva, Viladrich, Amado, González-Ponce, \& García-Calvo, 2014). En particular, se ha logrado establecer que aquellos estudiantes que poseen autoconcepto positivo mejoran sus calificaciones posteriores, independiente del rendimiento inicial (Miñano \& Castejón, 2011).

La percepción de competencia no solo mejora el rendimiento académico, sino que promueve otros resultados deseables, tales como: mayor compromiso con las tareas escolares, mayor perseverancia en tareas de mayor complejidad y mejor ajuste académico (Chase, Hilliard, Geldhof, Warren ,\& Lerner, 2014; Esnaola et al., 2008; Deci \& Ryan, 2013; Filak \& Sheldon, 2003; Kozan, Fabio, Blustein, \& Kenny, 2014; Madill, Gest, \& Rodkin, 2014; Ratelle \& Duchesne, 2014), mayor calidad en la ejecución de trabajos (Salum, Marín, \& Reyes, 2011), mayor motivación al logro (Carbonero et al., 2010; Deci \& Ryan, 2013; Filak \& Sheldon, 2003; Ratelle \& Duchesne, 2014) y mayor esperanza de éxito y menor ansiedad en pruebas estandarizadas (Muso, 2009; Urhahne, Chao, Florineth, Luttenberger, \& Paechter, 2011). Además, el autoconcepto presente en la etapa escolar tiene efectos a largo plazo, influenciando el desempeño académico en años posteriores, el nivel educativo alcanzado en la adultez (Guay et al., 2004) y en las aspiraciones profesionales futuras (Marsh \& Nagengest, 2012).

Los estudios sobre intervenciones destinadas a mejorar el rendimiento académico muestran al autoconcepto como una de las variables más efectivas por sobre otras variables cognitivo motivacionales, tales como las atribuciones causales, expectativas, metas, entre otras, además de tomar un papel moderador en el efecto de éstas variables sobre el rendimiento (Miñano, Cantero \& Castejón, 2008; Miñano \& Castejón, 2011).

Si se desea diseñar intervenciones para mejorar el autoconcepto, es necesario contar con instrumentos de medición confiables y válidos. En la medición del autoconcepto académico se han utilizado, principalmente, técnicas autodescriptivas (Anual, Bracho, Brito, Rondón, \& Sulbarán, 2012; Tomás \& Oliver,
2004) desarrollándose instrumentos tales como The Self Perception Inventory (Soares \& Soares, 1980, como se cita en González \& Touron, 1992), The Preschool and Primary Self Concept (Stager \& Young, 1982, citado en González \& Touron, 1992) y la Escala de Autoconcepto (AF5) (García \& Musitu, 1995, citado en Riquelme \& Bravo, 2011). Sin embargo, por su sustento empírico y enfoque multidimensional, el más utilizado ha sido el Academic Self Description Questionnaire (ASDQ) (Marsh, 1990) con sus dos versiones, ASDQ-I para $5^{\circ}$ y $6^{\circ}$ grados, y ASDQ-II, para $7^{\circ}$ a $10^{\circ}$ grados.

Las escalas existentes para medir el autoconcepto académico presentan diversas deficiencias para evaluar el progreso en programas de intervención. Las escalas ASDQ, aunque tiene una amplia utilización en distintas latitudes del mundo, son escalas muy extensas y poco funcionales para orientar una intervención del educador sobre comportamientos de la interacción profesor-alumno. El AF5, por su parte, aunque está validado en población chilena (García, Gracia, \& Seleznova, 2013) y ser breve, es una escala de autoconcepto global que captura de manera general la dimensión académica y no cubre los rangos etarios de los primeros niveles de escolaridad.

Una carencia importante en la investigación sobre el autoconcepto es la falta de atención a mediciones de las prácticas de estimulación por parte de los docentes. En diversas investigaciones y meta análisis sobre intervenciones realizadas desde la década del 90' con el objetivo de mejorar el autoconcepto de los estudiantes (BaenaExtremera \& Granero-Gallegos, 2013; Green et al., 2006; Haney \& Durlak, 1998; Hartie, 1992; O'Mara, Marsh, Craven, \& Debus, 2005, 2006; Marsh \& Martin, 2011) se observa que sólo se mide la mejora del autoconcepto académico de los estudiantes y no el grado en que los docentes lo estimulan. Esto evidencia la carencia de instrumentos que ayuden al docente a identificar su nivel de habilidad para estimular esta variable.

Seis meta-análisis han sido realizados sobre las intervenciones en el autoconcepto escolar, con la finalidad de esclarecer qué y cómo se puede mejorar esta variable. El primero de ellos fue realizado por Hartie en el año $1992(N=89)$, el segundo por Haney y Durlak en el año $1998(N=99)$ y un tercer meta análisis realizado por O'Mara et al. (2005; 2006), (N=145). El hallazgo más importante de estos estudios es la mayor efectividad de un enfoque multidimensional del autoconcepto por sobre aquel que lo concebía como un constructo unidimensional. Es decir, mientras más específica es la faceta del autoconcepto a mejorar, mayor es la probabilidad de éxito.

Green et al. (2006), realizan un cuarto meta análisis $(N=105)$, excluyendo a aquellas intervenciones que no se ejecutaban en los centros educativos. Corroboran los hallazgos de Craven y Debus, y señalan mayor efectividad cuando los programas de intervención son ejecutados por los propios docentes en lugar de profesionales 
externos. La modalidad intracurricular, en la intervención de las variables cognitivo motivacionales, ha demostrado mayor efectividad para mejorar los resultados académicos (Beaumont, Canning, \& Moscrop, 2016; Hui-Ju, 2009; Isiksal, 2010; Lobos \& Diaz, 2015; Marsh \& Martin, 2011; Tempelaar, Rienties, \& Nguyen, 2017).

Un quinto meta análisis lo realizan O'Mara et al. (2006), en donde centran su atención en las estrategias utilizadas para la modificación del autoconcepto académico. Al respecto concluyen que las intervenciones que involucraban la alabanza y las técnicas de retroalimentación resultaron ser mejores predictores del tamaño del efecto que otro tipo de estrategias utilizadas para la modificación del autoconcepto.

Entre todas las posibles intervenciones que pueden realizar los docentes, destaca el papel de la retroalimentación y el elogio (Lobos \& Díaz, 2015; Santana \& García, 2011; Shütze, Rakoczy, Klieme, Besser, \& Lesiss, 2017; Wollenschlager, Hattie, Machts, Möller, \& Harms, 2016). Se ha encontrado que mayores medidas de autoconcepto en los estudiantes están asociadas a un mayor nivel de competencias docentes para retroalimentar y elogiar adecuadamente (Olson \& Wyett, 2000; Santana \& García, 2011). El uso del elogio y mensajes instructivos podría incentivar en el estudiante un sentido de mayor competencia (Deci \& Ryan, 2013; Doménech \& Gómez, 2011; Lobos \& Díaz, 2015; Lobos, Díaz, Núñez y Pérez, 2015).

Finalmente, Huang (2011) realizó un meta análisis de las intervenciones en autoconcepto académico publicadas hasta el año $2007(N=278)$. Entre sus conclusiones plantea que los programas que incluían el desarrollo de habilidades académicas en conjunto con la estimulación del autoconcepto académico lograban ser más efectivos; las intervenciones que incluían uno solo de estos dos elementos, mostraban efectos más pequeños y de corto plazo.

Para estimular el autoconcepto es necesario conocer en qué ámbitos el docente puede usar la retroalimentación y el elogio para aportar percepciones particulares de competencia escolar en sus estudiantes (Deci \& Ryan, 2013; Doménech \& Gómez, 2011; Olson \& Wyett, 2000; Santana \& García, 2011; Shütze et al., 2017; Wollenschlager et al., 2016). En el ambiente de aula, se pueden distinguir tres situaciones específicas en las cuales el alumno presenta conductas susceptibles de ser retroalimentadas: cuando un estudiante participa en clases, cuando muestra el uso de procedimientos de trabajo y cuando demuestra capacidad adquirida en la ejecución de tareas escolares (Lobos et al., 2015).

En el último tiempo, se ha comenzado a enfatizar la necesidad de medir fidelidad del tratamiento en la intervención con estudiantes (Campbell et al., 2015; Bruhn, Hirsch, \& Lloyd, 2015; Desimone \& Hill, 2017; Grandstaff-Beckers, Saal, \& Cheek, 2013; Schütze, Rakoczy, Klieme, Besser, \& Leiss, 2017; Sutherland,
Conroy, McLeod, Algina, \& Wu, 2017), es decir la importancia del nivel de competencia y adhesión del docente al momento de utilizar prácticas basadas en la evidencia. Para que esto sea factible se requiere contar con instrumentos que evalúen el grado en que los profesores manejan las estrategias, es este caso, su nivel de habilidad para estimular el autoconcepto de los estudiantes, de manera que el docente pueda conocer y considerar esa percepción para influir deliberadamente sobre ella en los programas de intervención.

Por lo tanto, el propósito de este estudio es diseñar y evaluar el comportamiento psicométrico de la Escala de Estrategias de Estimulación del Autoconcepto Académico, asociada a comportamientos observables de la interacción profesor-alumno. En particular, se espera identificar y confirmar la estructura factorial del instrumento, determinar su consistencia interna, e indicadores de validez concurrente con una escala de autoestima escolar académica.

\section{Método}

Este estudio corresponde a un diseño instrumental, que se caracteriza por utilizar múltiples metodologías para desarrollar pruebas psicométricas. Esta investigación forma parte de un estudio mayor, correspondiente a un estudio cuasi-experimental sobre el efecto de un programa de entrenamiento en estrategias de estimulación del autoconcepto académico (Lobos, 2015).

\section{Participantes}

La población corresponde a estudiantes de educación primaria entre 7 y 10 años, de escuelas insertas en contexto de pobreza, que obtienen puntuaciones inferiores a la media nacional en pruebas estandarizadas del SIMCE, Sistema de Medición de la Calidad Educativa (Agencia de Calidad de la Educación Gobierno de Chile, 2015), de la Provincia de Concepción, Chile.

Un total de 819 estudiantes, de entre 8 a 10 años, de tercero y cuarto año básico de 16 establecimientos participaron en el estudio. De ellos, se eliminaron 7 por falta de respuesta en sus cuestionarios, siendo la muestra final de 812 participantes. Los alumnos evaluaron a un total de 36 profesores, los cuales recibieron en promedio 22.56 evaluaciones $(D E=7.82)$. Un $50.5 \%$ de los participantes fueron mujeres y la edad promedio de los alumnos fue de 9.09 años $(D E=1.05)$. El muestreo fue realizado de modo no probabilístico, de manera intencionada; las administraciones municipales definen los grupos en los que se autoriza investigar, principalmente utilizando el criterio de no estar participando en otro programa de investigación. Se encuestaron a los cursos completos, sin selección de participantes.

Se dividió a la muestra total en dos partes, estratificando las muestras por pertenencia al grupo control o experimental del estudio original y centro educativo. En 
la primera mitad se realizaron los análisis factoriales de carácter exploratorio $(n=407)$ y en la segunda mitad, se realizaron los análisis factoriales de tipo confirmatorio $(n=405)$. La distribución de edad, sexo y pertenencia a grupo control y experimental se puede observar en la Tabla 1.

Tabla 1

Muestra para Análisis Factorial Exploratorio y Confirmatorio

\begin{tabular}{lcccc}
\hline & \multicolumn{2}{c}{ Muestra 1 (AFE) } & \multicolumn{2}{c}{ Muestra 2 (AFC) } \\
\hline $\mathrm{N}$ & 407 & & 405 & $(\mathrm{DE}=1.06)$ \\
Edad & 9.06 & $(\mathrm{DE}=1.04)$ & 9.13 & $52 \%$ \\
Mujeres & 191 & $49 \%$ & 198 & $48 \%$ \\
Hombres & 199 & $51 \%$ & 183 & $46 \%$ \\
Grupo Control & 187 & $46 \%$ & 188 & $54 \%$ \\
Grupo Experimental & 220 & $54 \%$ & 217 & \\
\hline
\end{tabular}

\section{Instrumento}

La Escala de Estrategias de Estimulación del Autoconcepto Académico, en su forma final, está compuesta por 15 ítems. Se solicita a los estudiantes que declaren en qué medida considera que la afirmación se asemeja al comportamiento del profesor ante sus desempeños en el aula, en relación con las estrategias para estimular el autoconcepto en las dimensiones de Capacidad, Procedimiento de Trabajo y Participación en las Clases. Cada ítem se responde con una escala tipo Likert de seis puntos, donde 1 significa "nunca" y 6 significa "siempre". Las tres subescalas son:

Subescala de Estrategias de Estimulación de la Capacidad. Consiste en expresar al estudiante, con precisión, desempeños que denotan habilidad, mediante mensajes instructivos acompañados de elogio.

Subescalas de Estrategias de Estimulación del Procedimiento de Trabajo. Se refiere a manifestar elogios indicando las formas de trabajo en el aula como mantener el orden de útiles, seguir instrucciones, prestar atención, etc.

Subescala de Estrategias de Estimulación de la Participación en Clases. Incluye elogiar comportamientos de preguntar, comentar, responder y aclarar dudas.

Además de la Escala de Estrategias de Estimulación del Autoconcepto Académico, se aplicó la Escala de Autoestima Escolar Académica del Inventario de Autoestima de Coopersmith, validado para población chilena (Brinkmann, Segure, \& Solar, 1989). Esta escala está compuesta por 8 ítems, en los cuales el sujeto lee una afirmación sobre su persona relacionada al ámbito escolar y declara si ésta es "igual que yo" o "distinto a mî". Cada ítem con respuesta positiva es evaluada con 2 puntos.

\section{Procedimiento}

\section{Construcción de Instrumento}

Se elaboraron 32 reactivos, basados en la revisión de literatura y con la intención de disponer de un repertorio suficiente de preguntas para realizar una selección posterior de los mejores ítems. Los ítems fueron construidos considerando tres dimensiones, pertinentes a las tres situaciones en que la conducta de los alumnos puede ser retroalimentada: Participación, Capacidad y Procedimiento. Se estudió la validez de contenido mediante el juicio de expertos, seleccionando seis profesionales, bajo dos criterios: 1. grado de Magister y/o Doctor, y 2. producción científica vinculada a la Psicología Educacional.

Del proceso anterior, se eligieron 14 ítems que fueron aplicados en forma piloto a 44 estudiantes, con el fin de evaluar la claridad de las instrucciones y la pertinencia del lenguaje. La principal dificultad detectada fue la baja precisión en las indicaciones para responder a los reactivos, por lo que fue modificada remplazando la frase "selecciona el comportamiento..." por "indique la frecuencia del comportamiento...”. Al aplicar esta versión a una segunda muestra piloto de 161 alumnos, se eliminaron tres ítems que generaban un factor independiente, atribuible a su redacción invertida. Esta versión del instrumento quedó con cuatro ítems para Capacidad, 4 ítems para Procedimiento y tres para Participación. Se decidió reformular todos los reactivos en búsqueda de mayor precisión del constructo, y se agregaron tres ítems en Participación, cinco en Procedimiento y 1 en Capacidad, para aumentar la frabilidad de las escalas.

Se realiza un tercer piloto en una muestra de 288 participantes, con el instrumento compuesto por 20 ítems (cinco ítems en Capacidad, 9 en Procedimiento y seis en participación). Tras realizar análisis factorial exploratorio y análisis de fiabilidad de las escalas, se decide eliminar cuatro ítems de la escala de Procedimiento, por cargas factoriales bajas, y cuatro ítems de la escala de Participación porque presentaban cargas cruzadas importantes con Procedimiento de trabajo. Para mantener la confiabilidad de la escala de Participación, se crearon cuatro ítems nuevos. 
Tras definir la estructura final del instrumento, se organizó su aplicación en distintos establecimientos educacionales, obteniéndose una nueva muestra para este estudio de 819 estudiantes. La coordinación para las aplicaciones del instrumento fue desarrollada en forma directa con las escuelas, previa autorización y derivación de las administraciones comunales.

En el caso de los estudiantes, se les aplicó a los directores y apoderados (padres y cuidadores) el protocolo de consentimiento informado consignado por firma de acta de consentimiento. Además, los estudiantes fueron invitados a participar voluntariamente, usando la modalidad de asentimiento informado y asegurando la confidencialidad de la información entregada. El proyecto fue sometido a revisión en sus aspectos éticos por un comité académico del Programa de Doctorado en Psicología, de la Facultad de Ciencias Sociales, de la Universidad de Concepción, Chile.

La aplicación de instrumentos fue realizada por los investigadores del presente estudio y encuestadores previamente entrenados. La aplicación de instrumentos tuvo una duración de 30 minutos aproximadamente.

\section{Análisis de Datos}

Análisis Factorial Exploratorio (AFE). Se analizaron los ítems de forma descriptiva, para detectar ítems que fuesen problemáticos, ya sea por escasa variabilidad o por su asimetría o curtosis extrema que dificultasen su análisis posterior. El carácter ordinal de los ítems llevó al uso de la matriz de correlaciones policóricas para los análisis posteriores. Se probó la adecuación de la matriz al análisis factorial utilizando el test de Identidad de Barttlet, así como el indicador KMO.

Considerando el resultado del análisis paralelo de Horn y la existencia de una estructura teórica previa, se fijó el número de factores a tres, correspondientes a las dimensiones de Procedimiento, Capacidad y Participación a partir de las cuales se construyeron los ítems. La extracción de factores sobre la matriz de correlaciones policóricas se realizó usando mínimos cuadrados. La rotación de la solución extraída fue de tipo Procrusto, que implica el ajuste de la solución factorial a una solución predeterminada; en este caso, se busca obtener una matriz en la cual las cargas factoriales mayores de los ítems se presente en el factor al cual está asignado, y sea cero en los otros factores.

A partir de los resultados preliminares, se eliminaron los ítems que presentan cargas factoriales menores a 0.3 o cargas cruzadas y se volvió a realizar el procedimiento hasta obtener una solución satisfactoria.

Se estudió la confiabilidad de las subescalas resultantes como consistencia interna, mediante alfa de Cronbach, tanto de la matriz de varianza/covarianza original como la matriz de correlaciones policóricas. Considerando la existencia de una alta relación entre los factores, se estudió la confiabilidad de una medida general usando omega jerárquico con la misma rotación Procrusto utilizada previamente.

Análisis Factorial Confirmatorio (AFC). Se realizó un análisis confirmatorio utilizando lavaan con estimación WLSMV por ser recomendada para muestras de pequeño a moderado tamaño, sin necesidad de normalidad de las variables, y para nivel ordinal de medición (Hair, Black, Babin, \& Anderson, 2009). Como indicadores de medida de ajuste del modelo se utilizaron $\chi^{2}$ absoluto y normalizado, CFI, TLI y RMSEA; cómo valores de corte para considerar los modelos como apropiados, se utilizó CFI y TLI superiores a 0.95, y RMSEA cercano o inferior a 0.05 (Williams \& O'Boyle, 2011). Se modeló la solución considerando un modelo de segundo orden. Se comparó el ajuste del modelo de segundo orden con un modelo unidimensional, para determinar si era apropiado simplificar el modelo eliminando los factores primarios.

Validez de Criterio. Para estudiar la validez de criterio concurrente del instrumento se calculó la correlación rho de Spearman de la puntuación general de la escala de Estrategias de Estimulación del Autoconcepto Académico con la Sub Escala de Autoestima Escolar Académica (EAEA) del Inventario de Autoestima de Coopersmith, validado para población chilena (Brinkmann et al., 1989).

\section{Resultados}

\section{Análisis Factorial Exploratorio}

En la Tabla 2 se presentan los estadísticos descriptivos para los ítems de la muestra exploratoria $(n=407)$. Se puede observar que las medias de los ítems se encuentran en el rango entre 4.05 y 4.84, indicando que la frecuencia de las conductas estudiadas se encuentra entre las categorías "Frecuentemente" y "Casi Siempre". La desviación estándar de los ítems es bastante similar, en el rango entre 1.52 y 1.88 , y la asimetría y curtosis de todos los ítems se encuentra en el rango entre -1.5 y 1.5 , lo que nos indica que no es necesario eliminar ítems por baja variabilidad o formas extremas que dificulten los análisis posteriores.

El resultado del test de Bartlett indicó que la matriz de correlaciones policóricas no proviene de una matriz identidad, $\chi^{2}(120)=1168.6, p<0.001$. Además, el índice $\mathrm{KMO}=0.95$ indicó que la matriz de correlaciones es apta para el análisis factorial.

El análisis paralelo de Horn sobre 5000 remuestras indica retener tres factores, lo cual coincide con la estructura de tres factores planteada teóricamente. La solución de tres factores para 16 ítems predice un 66\% de la varianza; el factor de Capacidades explica un $23 \%$ de la varianza, el de Participación un $23 \%$ y el de procedimiento el $20 \%$. Todos los ítems presentan cargas superiores a 0.3 en el factor asignado teóricamente e inferiores a este valor en los otros factores, excepto el ítem 13, "Mi profesora me felicita por la forma en que hago las actividades 
en clase", que presenta carga cruzada en los factores para Participación y Procedimiento.

Se prueba una segunda solución factorial, eliminado el ítem 13. Esta solución de15 ítems predice un $66 \%$ de la varianza; el factor de Capacidad explica un
$25 \%$ de la varianza, el de Participación un 23\% y el de Procedimiento el $18 \%$. En esta solución, la carga factorial mínima para un ítem en su factor asignado es de 0.47 y no se presentan cargas cruzadas. La solución factorial encontrada se presenta en la Tabla 3.

Tabla 2

Descriptivos Ítems Muestra Análisis Factorial Exploratorio

\begin{tabular}{|c|c|c|c|c|}
\hline Ítem & $M$ & $D E$ & Asimetría & Curtosis \\
\hline \multicolumn{5}{|l|}{ Capacidad } \\
\hline 1. Mi profesora me dice que aprendo con facilidad las materias. & 4,30 & 1,76 & $-0,67$ & $-0,94$ \\
\hline 2. Mi profesora me dice que soy inteligente para aprender. & 4,23 & 1,79 & $-0,60$ & $-1,07$ \\
\hline 3. Mi profesora me dice que soy capaz para los estudios. & 4,52 & 1,73 & $-0,85$ & $-0,66$ \\
\hline 4. Mi profesora me dice que tengo habilidad para aprender. & 4,33 & 1,76 & $-0,72$ & $-0,90$ \\
\hline 5. Mi profesora me expresa que soy bueno o buena para aprender. & 4,42 & 1,76 & $-0,80$ & $-0,77$ \\
\hline \multicolumn{5}{|l|}{ Participación } \\
\hline 6. Mi profesora me expresa su agrado por mi participación en clases. & 4,41 & 1,82 & $-0,78$ & $-0,88$ \\
\hline 7. Mi profesora me felicita por los comentarios que hago en clases. & 4,05 & 1,88 & $-0,49$ & $-1,27$ \\
\hline 8. Mi profesora me expresa su agrado por dar opiniones en clases. & 4,25 & 1,82 & $-0,60$ & $-1,12$ \\
\hline 9. Mi profesora me expresa su agrado cuando respondo las preguntas que hace. & 4,38 & 1,69 & $-0,63$ & $-0,96$ \\
\hline 10. Mi profesora me felicita cuando yo le digo mis dudas en su clase. & 4,17 & 1,88 & $-0,56$ & $-1,20$ \\
\hline 11. Mi profesora me felicita cuando participo en clases. & 4,73 & 1,67 & $-1,10$ & $-0,19$ \\
\hline \multicolumn{5}{|l|}{ Procedimiento } \\
\hline 12. Mi profesora me felicita cuando termino los trabajos en el tiempo indicado. & 4,67 & 1,63 & $-0,98$ & $-0,33$ \\
\hline 13. Mi profesora me felicita por la forma en que hago las actividades en clase. & 4,55 & 1,65 & $-0,86$ & $-0,57$ \\
\hline 14. Mi profesora me felicita cuando yo termino mis trabajos en clase. & 4,84 & 1,52 & $-1,13$ & 0,04 \\
\hline 15. Mi profesora me felicita cuando yo sigo las instrucciones que ella me ha dado en la clase. & 4,77 & 1,60 & $-1,10$ & $-0,09$ \\
\hline 16. Mi profesora me felicita cuando hago con cuidado las actividades de la clase. & 4,74 & 1,64 & $-1,10$ & $-0,13$ \\
\hline
\end{tabular}

Tabla 3

Solución Factorial para Muestra de Análisis Factorial Exploratorio

\begin{tabular}{|c|c|c|c|c|}
\hline Ítem & CAP & PAR & PROC & Estadísticos \\
\hline \multicolumn{5}{|l|}{ Capacidad } \\
\hline Ítem 1 & 0,67 & 0,28 & $-0,18$ & $M=4.36$ \\
\hline Ítem 2 & 0,83 & $-0,04$ & 0,07 & $D E=1.44$ \\
\hline Ítem 3 & 0,64 & 0,07 & 0,11 & $\alpha=.88$ \\
\hline Ítem 4 & 0,91 & $-0,08$ & 0,02 & $\alpha_{p}=.91$ \\
\hline Ítem 5 & 0,89 & $-0,09$ & 0,07 & \\
\hline \multicolumn{5}{|l|}{ Participación } \\
\hline Ítem 6 & 0,16 & 0,82 & $-0,16$ & $M=4.33$ \\
\hline Ítem 7 & $-0,06$ & 0,85 & 0,00 & $D E=1.41$ \\
\hline Ítem 8 & 0,09 & 0,75 & $-0,07$ & $\alpha=.88$ \\
\hline Ítem 9 & 0,11 & 0,47 & 0,22 & $\alpha_{p}=.91$ \\
\hline Ítem 10 & $-0,06$ & 0,73 & 0,13 & \\
\hline Ítem 11 & $-0,09$ & 0,74 & 0,19 & \\
\hline \multicolumn{5}{|l|}{ Procedimiento } \\
\hline Ítem 12 & 0,03 & 0,02 & 0,81 & $M=4.33$ \\
\hline Ítem 14 & 0,04 & 0,14 & 0,68 & $D E=1.41$ \\
\hline Ítem 15 & 0,10 & 0,06 & 0,68 & $\alpha=.88$ \\
\hline Ítem 16 & 0,08 & 0,07 & 0,68 & $\alpha_{p}=.89$ \\
\hline
\end{tabular}

Nota. CAP: Factor Capacidad. PAR: Factor Participación. PROC: Factor Procedimiento. $\alpha$ : alfa de Cronbach basado en la matriz de varianza/covarianza en bruto. $\alpha_{\mathrm{p}}$ : alfa de Cronbach basado en la matriz de correlaciones policóricas 
La correlación entre los factores de la solución es fuerte; la correlación entre Capacidad y Participación es de $r=.81$, entre Capacidad y Procedimiento es de $r=.72$ y entre Participación y Procedimiento es de $r=.79$. Estas correlaciones altas y de similar tamaño nos permiten inferir la presencia de un factor de segundo orden, que explica la variabilidad común de todos los ítems. Si se calcula este factor general, presenta una media de 4.46 (DE $=1.25$ ), un alfa de Cronbach de .94, un alfa policórico de .95 y un omega jerárquico de .89 .

\section{Análisis Factorial Confirmatorio}

Se realizó un análisis factorial confirmatorio de la solución de 15 ítems sobre la segunda mitad de la muestra $(n=405)$. Se utilizó un modelo factorial de segundo orden, que considera que los factores de Capacidad, Participación y Procedimiento dependen de un factor general de uso de estrategias de estimulación del autoconcepto académico.

Al analizar los datos, se observa una diferencia estadísticamente significativa entre el modelo y los datos $\chi^{2}(87)=148.09, p<0.001$. Sin embargo, los indicadores de ajuste relativo son buenos, ya que el $\chi^{2}$ normalizado es igual a 1.7, inferior al valor de 3 recomendado; $\mathrm{CFI}=.99$ y TLI $=.99$ están por sobre el .95 recomendado; y RMSEA $=.046, p=.676$ no es estadísticamente significativo y menor a 0.05 . La estructura factorial de esta solución se puede observar en la Figura 1.

Se verificó si la solución unifactorial era un modelo apropiado para los datos. Este modelo no se ajusta de forma a absoluta a los datos, $\chi^{2}(90)=243.1, p<0.001$. Sus índices de ajuste relativo son adecuados, aunque no óptimos, ya que se observa un $\chi^{2}$ normalizado de 2.7, apenas inferior al valor de 3 recomendado; CFI $=.95 \mathrm{y}$ $\mathrm{TLI}=.94$ que están muy cerca del .95 recomendado; y RMSEA $=.072, p=.001$, que es superior a 0.05 y estadísticamente significativo. Al comparar el modelo factorial de segundo orden con el modelo unidimensional usando la prueba de razón de verosimilitud, se observa que este último presenta un ajuste a los datos peor que el modelo de segundo orden, siendo esta diferencia estadísticamente significativa, $\chi^{2}(2.43)=67.21, p<0.001$.

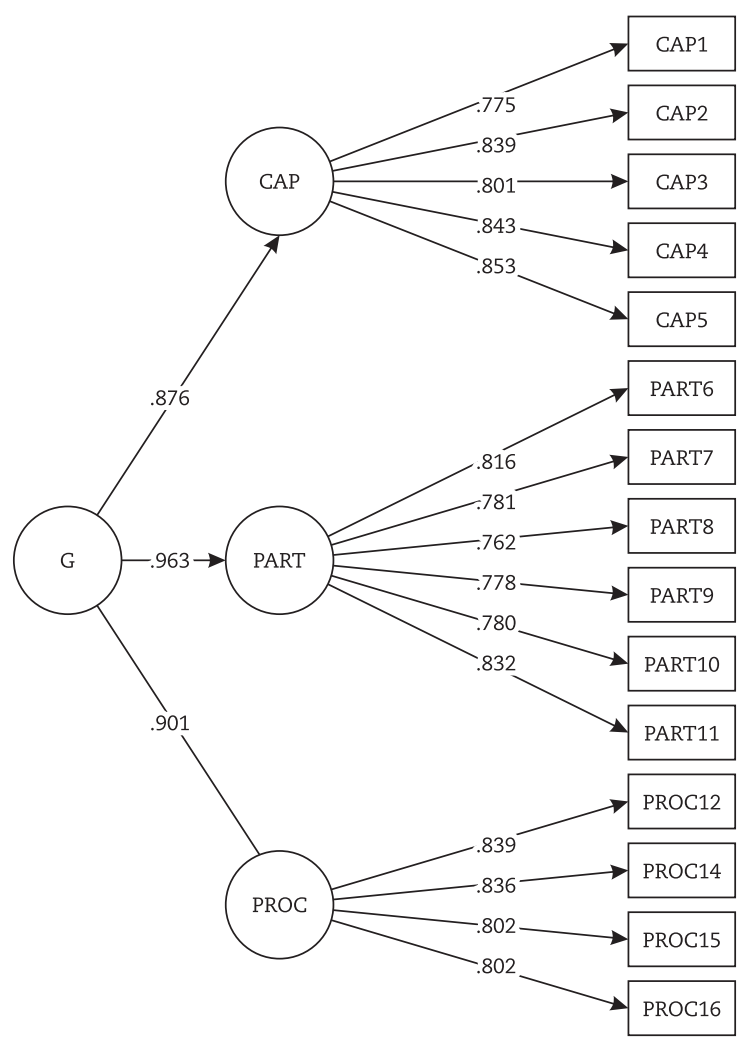

Figura 1. Solución factorial para muestra de análisis factorial confirmatorio

\section{Análisis de Validez Concurrente}

En la muestra confirmatoria, se observa una correlación moderada entre las puntuaciones del instrumento en estudio y la Sub Escala de Autoestima Escolar de Coopersmith (utilizando el coeficiente de correlación por rango de Spearman), siendo positivas y significativas, $r_{s}=0.24, p<0.001$. Esto es, a mayor reporte del estudiante 
de la aplicación de estrategias de estimulación del autoconcepto por parte de su profesor, mejor es la autoestima escolar académica del estudiante.

\section{Discusión}

El objetivo de este estudio es diseñar y evaluar el comportamiento psicométrico de la Escala de Estrategias de Estimulación del Autoconcepto Académico, asociada a comportamientos observables de la interacción profesor-alumno. Se identificó teóricamente una estructura de tres factores (Capacidad, Participación y Procedimiento), la cual fue validada por expertos y se ajusta a los resultados del análisis factorial exploratorio. Considerando la fuerte relación entre los factores, se postula una estructura factorial de segundo orden, con un factor general de estimulación del autoconcepto relacionado fuertemente con estos tres factores de primer orden. Se observa una alta consistencia interna de los tres factores de primer orden, así como del factor general. La estructura factorial propuesta por el análisis factorial exploratorio se corroboró mediante análisis factorial confirmatorio, encontrándose buenos indicadores de ajuste. Finalmente, existen indicadores de validez concurrente con la escala de autoestima escolar académica de Coopersmith.

La estructura factorial jerárquica de segundo orden puede ser justificada teóricamente. El factor general, con una alta correlación con los tres factores primarios, puede estar asociado a la disposición o preocupación del docente por los ritmos de aprendizaje de sus estudiantes (Castro, Paternina, \& Gutiérrez, 2014; Mena, Bugueño, \& Valdés, 2015; Robledo, 2011; Tuc, 2013), que le permite estimular el autoconcepto de los alumnos en el momento indicado. Esta disposición es transversal a todas las estrategias específicas, mediante el uso de mensajes instructivos y elogio. Ahora bien, este factor general no puede explicar por completo el uso de estrategias de estimulación del autoconcepto por parte de los docentes, dado que cada dimensión (capacidad, participación y procedimiento), aluden a conductas que demandan del docente capacidades distintas. De esta manera, las estrategias de estimulación de la capacidad requieren que el docente deduzca qué competencias cognitivas utiliza el estudiante al ejecutar una actividad pedagógica; en cambio, las estrategias de estimulación del procedimiento de trabajo, se formulan sobre la adecuación de la conducta del estudiante a parámetros previamente definidos, explícitos y observables. Finalmente, las estrategias referidas a la participación dependen de la capacidad del docente para integrar los aportes verbales del estudiante a los contenidos impartidos en aula (Lobos, 2015).

La principal limitación del estudio fue el uso de un procedimiento de muestreo no probabilístico, lo que representa una limitación para la generalización de los resultados a la población. Los estudiantes seleccionados pertenecen solo a escuelas públicas por lo que es necesario estudiar cómo se comporta esta escala en otros contextos.

En conclusión, se aporta una escala compuesta por 15 reactivos, dirigida a población escolar básica, de habla hispana, diseñada y validada en idioma español, con indicadores tanto de validez de contenido, como dimensional y concurrente con autoestima escolar académica. Es una escala de fácil y sencilla aplicación, que permite a un profesor definir aspectos específicos de su interacción con los estudiantes, que favorecen el autoconcepto académico, lo que puede ser útil para desarrollar y evaluar programas de formación docente.

\section{Referencias}

Agencia de Calidad de la Educación Gobierno de Chile. (2015). Informe técnico SIMCE 2013. Retrieved from encurtador.com.br/iFVZ2

Anual, A., Bracho, A., Brito, N., Rondón, J. E., \& Sulbarán, D. (2012) Autoacepctación y mecanismos cognitivos sobre la imagen corporal. Psicothema, 24(3), 390-395. Recuperado de http://www.psicothema.es/pdf/4028.pdf

Baena-Extremera, A., \& Granero-Gallegos, A. (2013) Efecto de un programa de Educación de Aventura en la orientación al aprendizaje, satisfacción y autoconcepto en secundaria. Revista Iberoamericana de Diagnóstico y Evaluación Psicológica, 2(36), 163-182. Recuperado de http://www.redalyc.org/articulo.oa?id=459645436009

Beaumont, C., Canning, S., \& Moscrop, C. (2016). Easing the transition from school to HE: scaffolding the development of self- regulated learning through a dialogic approach to feedback. Journal of Further and Higher Education, 40(3), 331-350. doi: 10.1080/0309877X.2014.953460

Brinkmann, H., Segure, T., \& Solar, M. I. (1989). Adaptación y estandarización del Inventario de Autoestima de Coopersmith. Revista Chilena de Psicología, 10(1), 73-87. Recuperado de http://www2.udec.cl/ hbrinkma/articulo_coopersmith.pdf

Bruhn, A., Hirsch, S., \& Lloyd, J. (2015). Treatment integrity in school-wide programs: A review of the literature (1993-2012). The Journal of Primary Prevention, 36(5), 335-349. doi: 10.1007/s10935-015-0400-9

Campbell, R., Rawlins, E., Wells, S., Kipping, R., Chittleborough, C., Peters, T., Lawlor, D., \& Jago, R. (2015). Intervention fidelity in a school-based diet and physical activity intervention in the UK: Active for Life Year 5. International Journal of Behavioral Nutrition and Physical Activity, 12(1), 2-14. doi: 10.1186/s12966-015-0300-7

Carbonero, M., Martín-Antón, L., Reoyo, N., \& Román, J. (2010). Efecto de un programa de entrenamiento al profesorado en la motivación, clima de aula y estrategias de aprendizaje de su alumnado. Revista Iberoamericana de Psicología y Salud, 1(2), 117-138. Recuperado de http://www.redalyc.org/html/2451/245116406001/ 
Castejón, J., \& Miñano, P. (2011). Variables cognitivas y motivacionales en el rendimiento académico en Lengua y Matemáticas: Un modelo estructural. Revista de Psicodidáctica, 16(2), 203-230. Recuperado de http://www.redalyc.org/pdf/175/17518828003.pdf

Castro, S., Paternina, A., \& Gutiérrez, M. (2014). Factores pedagógicos relacionados con el rendimiento académico en estudiantes de cinco instituciones educativas del distrito de Santa Marta, Colombia. Revista Intercontinental de Psicología y Educación, 16(2), 151-169. Recuperado de http://www.redalyc.org/articulo.oa?id=80231541009

Chase, P. A., Hilliard, L. J., Geldhof, G. J., Warren, D. J. A., \& Lerner, R.M. (2014). Academic achievement in the high school years: The changing role of school engagement. Journal of Youth and Adolescence, 43(6), 884-896. doi: 10.1007/s10964-013-0085-4

Coelho, V., Sousa, V., \& Figueira, A. (2014). The impact of a school-based social and emotional learning program on the self-concept of middle school students. Revista de Psicodidáctica, 19(2), 347-365. doi: 10.1387/RevPsicodidact.10714

Desimone, L., \& Hill, K. (2017). Inside the black box: Examining mediators and moderators of a middle school science intervention. Educational Evaluation and Policy Analysis, 39(3), 511-536. doi: 10.3102/0162373717697842

Deci, E., \& Ryan, R. (2013). Toward a social psychology of assimilation: Self-determination theory in cognitive development and education. In B. W. Sokol, F. M. E. Grouzet \& U. Muller (Eds.), Self-regulation and autonomy: Social and developmental dimensions of human conduct (pp. 191-207). New York: Cambridge University Press.

Doménech, F., \& Gómez, A. (2011). Relaciones entre las necesidades psicológicas del estudiante, los enfoques de aprendizaje, las estrategias de evitación y el rendimiento. Electronic Journal of Research in Educational Psychology, 9(24) 463-496. Recuperado de http://www. investigacion-psicopedagogica.org/revista/articulos/24/english/Art_24_597.pdf

Esnaola, J., Goñi, A., \& Madariaga, J. (2008). El autoconcepto: Perspectivas de investigación. Revista Psicodidáctica, 13(1), 69-96. Recuperado de http://www.redalyc.org/pdf/175/17513105.pdf

Filak, V., \& Sheldon, K. (2003). Student psychological need satisfaction and college teacher-course evaluation. Educational Psychology, 23(3), 235-247. doi: 10.1080/0144341032000060084

García, F., Gracia, E., \& Seleznova, A. (2013). Validation of the english version of the Five Factor Self-concept Cuestionnarie. Phicothema, 25(4), 549-595. doi: 10.7334/psicothema2013.33

Gallardo, B., Garfella, P., Sánchez, F., Ros, C., \& Serra, B. (2008). La influencia del autoconcepto en el rendimiento académico de estudiantes universitarios. Revista Española de Orientación y Psicopedagogía, 20(1), 16-28. Recuperado de https://www2.uned.es/reop/ pdfs/2009/20-1\%20-\%20Bernardo\%20Gargallo.pdf

González, J., Núñez, J., Glez, S., \& García, M. (1997). Autoconcepto, autoestima y aprendizaje escolar. Psicothema, 9(2), 271-289. Recuperado de http://www.psicothema.com/pdf/97.pdf

González, M., \& Touron, J. (1992). Autoconcepto y rendimiento escolar: Sus implicaciones en la motivación y autorregulación en el aprendizaje. Pamplona: Ediciones Universidad de Navarra S.A.

Grandstaff-Beckers, G., Saal, L., \& Cheek, E. (2013). Investigating treatment fidelity and social validity of a peer-mediated postsecondary reading intervention. Reading Psychology, 34(4), 336-354. doi: 10.1080/02702711.2011.636477

Green, J., Marsh, H., \& O'Mara, A. (2006). Administering self-concept interventions in schools: No training necessary? A meta-analysis. International Education Journal, 7(4), 524-533. Recuperado de https://files.eric.ed.gov/fulltext/EJ854308.pdf

Guay, F., Boivin, M., \& Marsh, H. (2003). Academic self-concept and academic achievement: Developmental perspectives on their causal ordering. Journal of Educational Psychology, 95(1), 124-136. doi: 10.1037/0022-0663.95.1.124

Guay, F., Larose, S., \& Boivin, M. (2004). Academic self-concept and educational attainment level: A ten-year longitudinal study. Journal of Educational Psychology, 97(2), 78-91. doi: 10.1080/13576500342000040

Hair, J., Black, W., Babin, B., \& Anderson, R. (2009). Multivariate Data Analysis (7a ed.). Upper Saddle River, NJ: Prentice Hall.

Hartie, J. (1992). Self-concept. Hillsdale NJ: Lawrence Erlbaum.

Haney, P., \& Durlak, J. A. (1998). Changing self-esteem in children and adolescents: a meta-analytic review. Journal of Clinical Child Psychology, 27(4), 423-433. doi: 10.1207/s15374424jccp2704_6

Huang, C. (2011). Self-concept and academic achievement: A meta-analysis of longitudinal relations. Journal of School Psychology, 49(5), 505-528. doi: 10.1016/j.jsp.2011.07.001

Hui-Ju, L. (2009). Exploring changes in academic self-concept in ability-grouped english classes. Journal of Humanities and Social Sciences, 2(2), 411-432. Recuperado de http://cgjhsc.cgu.edu.tw/data_files/2-2\%2007.pdf

Isiksal, M. (2010). A comparative study on undergraduate students' academic motivation and academic self-concept. The Spanish Journal of Psychology, 13(2), 572-585. doi:10.1017/S1138741600002250

Kozan, S., Fabio, A. D., Blustein, D. L., \& Kenny, M. E. (2014). The role of social support and work-related factors on the school engagement of Italian high school students. Journal of Career Assessment, 22(2), 345-354. doi: 10.1177/1069072713493988

Lobos, K. (2015). Prácticas valorativas pedagógicas y autoconcepto académico (Tesis doctoral). Universidad de Concepción, Concepción, Chile.

Lobos, K. \& Díaz, A. (2015). Prácticas valorativas pedagógicas y autoconcepto (Tesis doctoral). Universidad de Concepción, Chile.

Lobos, K., Díaz, A., Bustos, C., \& Pérez, M.V. (2015). Construction and psychometric characteristics of the Self-Concept Scale of Interaction in the Classroom, Psicothema, 27(2), 152-158. doi: 10.7334/psicothema2014.224

Marsh, H. (1990). The causal ordering of academic self-concept and academic achievement: A multivariate, longitudinal panel analysis. Journal of Educational Psychology, 82(4), 646-656. http://bit.do/ex2LG

Marsh, H., \& Craven, R. (2006). Reciprocal effects of self- concept and performance from a multidimensional perspective. Perspectives on Psychological Science, 1(2), 133-163. doi: 10.1111/j.1745-6916.2006.00010.x

Marsh, H., \& Martin, A. (2011). Academic self-concept and academic achievement: Relations and causal ordering. British Journal of Educational Psychology, 81(1), 59-77. doi: 10.1348/000709910X503501

Marsh, H., \& Nagengest, B. (2012). Big fish in little ponds aspire more: Mediation and cross-cultural generalizability of school-average ability effects on self-concept and career aspirations in science. Journal of Educational Psychology, 104(4), 1033- 1053. doi: 10.1037/ a0027697

Madill, R. A., Gest, S. D., \& Rodkin, P. C. (2014). Students' perceptions of relatedness in the classroom: The roles of emotionally supportive teacher-child interactions, children's aggressive-disruptive behaviors, and peer social preference. School Psychology Review, 43(1), 86105. Recuperado de https://files.eric.ed.gov/fulltext/ED519001.pdf

Mena, I., Bugueño, X., \& Valdés, A. (2015). Vínculo pedagógico positivo: Principios para su desarrollo. Santiago de Chile: Universidad Católica. $2^{\mathrm{a}}$ ed. 
Miñano, P., \& Castejón, J. (2011). Variables cognitivas y motivacionales en el rendimiento académico en Lengua y Matemáticas: Un modelo estructural. Revista de Psicodidáctica, 16(2), 203-230. Recuperado de http://www.ehu.eus/ojs/index.php/psicodidactica/article/ view/930/1585

Miñano, P., Cantero, M., \& Castejón, J. (2008). Predicción del rendimiento escolar de los alumnos a partir de las aptitudes, el autoconcepto académico y las atribuciones causales. Horizontes Educacionales, 13(2), 11-23. Recuperado de http://www.redalyc.org/articulo. oa?id=97912401001

Muso, M. (2009). Evaluación de funciones ejecutivas en niños: Análisis y adaptación de pruebas en un contexto escolar. Revista Iberoamericana de Diagnóstico y Evaluación en Psicología, 27(1), 157-178. Recuperado de http://www.redalyc.org/html/4596/459645443009/

O'Mara, A., Marsh, H., Craven, R., \& Debus, R. (2005). Self-Concept intervention research in school settings: A multivariate, multilevel model metaanalysis. SELF Research Centre, University of Western Sydney, Australia.

O'Mara, A., Marsh, H., Craven, R., \& Debus, R. (2006). Do self-concept interventions make a difference? A synergistic blend of construct validation and meta-analysis, Educational Psychologist, 41(3), 181-206. doi: 10.1207/s15326985ep4103_4

Olson, C., \& Wyett, J. (2000). Teachers need affective competencies. Education, 120(4), 741-741. Retirado de http://findarticles.com/p/ articles/mi_qa3673/is_4_120/ai_n2878 4024/

Ratelle, C., \& Duchesne, S. (2014). Trajectories of psychological need satisfaction from early to late adolescence as a predictor of adjustment in school. Contemporary Educational Psychology, 39(4), 388-400. doi: 10.1016/j.cedpsych.2014.09.003

Riquelme, M., \& Bravo, P. (2011). Análisis Psicométrico confirmatorio de la medida multidimensional del Test de Autoconcepto Forma 5 en Español (AF5), en estudiantes Universitarios de Chile. Psicologia, Saúde \& Doenças, 12(1), 91-103. Recuperado de http://www.scielo. mec.pt/pdf/psd/v12n1/v12n1a06.pdf

Robledo, L., E. (2011). La transferencia en el proceso educativo. Revista Colombiana de Ciencias Sociales, 2(1), 85-89. Recuperado de http:// www.funlam.edu.co/revistas/index.php/RCCS/article/view/27/95

Salum, A., Marín, R., \& Reyes, C. (2011). Relevancia de las dimensiones del autoconcepto en estudiantes de escuelas secundarias de ciudad Victoria, Tamaulipas, México. Revista Electrónica de Psicología Iztacala, 14(2), 255-272. Recuperado de http://www.revistas.unam.mx/ index.php/repi/article/view/26037/24512

Sánchez-Oliva, D., Viladrich, C., Amado, D., González-Ponce, I. \& García-Calvo, T. (2014). Prediction of positive behaviors in physical education: A Self-Determination Theory perspective. Revista de Psicodidáctica, 19(2), 387-405. doi: 10.1387/RevPsicodidact.7911

Santana,L., \&García,L. (2011). Percepción de apoyodepadresyprofesores, autoconceptoy toma de decisionesen bachillerato. RevistadeEducación, 355, 493-519. https://www.mecd.gob.es/dctm/revista-de-educacion/articulosre355/re35521.pdf?documentId=0901e72b81202af1

Sutherland, K., Conroy, M., McLeod, B., Algina, J., \& Wu, E. (2017). Teacher competence of delivery of BEST in CLASS as a mediator of treatment effects. School Mental Health, 10(3), 214-225. doi: 10.1007/s12310-017-9224-5

Schütze, B., Rakoczy, K., Klieme, E., Besser, M., \& Leiss, D. (2017). Training effects on teachers' feedback practice: The mediating function of feedback knowledge and the moderating role of self-efficacy. ZDM, 49(3), 475-489. doi: 10.1007/s11858-017-0855-7

Tempelaar, D., Rienties, B., \& Nguyen, Q. (2017). Towards actionable learning analytics using dispositions. IEEE Transactions on Learning Technologies, 10(1), 6-16. doi: 10.1109/TLT.2017.2662679

Tomás, J., \& Oliver, A. (2004). Análisis psicométrico confirmatorio de una medida multidimensional del autoconcepto en español. Revista Interamericana de Psicología, 38(2), 285-293.

Tuc, M. (2013). Clima del aula y rendimiento escolar (Tesis de maestría inédita). Universidad Rafael Landívar, Quetzaltenango, Guatemala. Recuperado de http://www.scielo.mec.pt/scielo.php?script=sci_arttext\&pid=S1645-00862011000100006

Urhahne, D., Chao, S., Florineth, M., Luttenberger, S., \& Paechter, M. (2011). Academic self-concept, learning motivation and test anxiety of the underestimated student. British Journal of Educational Psychology, 81(1), 161-177. doi: 10.1348/000709910X504500

Williams, L. J., \& O'Boyle, E. (2011). The myth of global fit indices and alternatives for assessing latent variable relations. Organizational Research Methods, 14(2), 350-369. doi: 10.1177/1094428110391472

Wollenschläger, M., Hattie, J., Machts N., c, Möller, J. \& Harms, U. (2016). A What makes rubrics effective in teacher-feedback? Transparency of learning goals is not enough. Contemporary Educational Psychology 44-45, 1-11. doi: 10.1016/j.cedpsych.2015.11.003

\section{Sobre os autores}

Karla Lobos Peña es Psicóloga, Magister en Psicología Educativa y Doctor en Psicología, docente Facultad de Ciencias Sociales, Universidad Santo Tomás, Chile.

Alejandro Díaz Mujica es Psicólogo, Doctor Psicología y Director Doctorado en Psicología Universidad de Concepción, Chile.

Claudio Bustos Navarrete es Psicólogo, Magister en Estadística y docente Universidad de Concepción, Concepción, Chile.

Fabiola Sáez Delgado es Licenciada en Educación, Magister en Educación y docente Universidad de Concepción, Chile. 